\title{
A BRIEF REMARK ON BALANCING-WIEFERICH PRIMES
}

\author{
UTKAL KESHARI DUTTA, BIJAN KUMAR PATEL, and PRASANTA KUMAR RAY
}

\begin{abstract}
A prime $p$ is said to be a balancing-Wieferich prime if it satisfies the congruence $B_{p-\left(\frac{8}{p}\right)} \equiv 0\left(\bmod p^{2}\right)$, equivalently $\pi(p)=\pi\left(p^{2}\right)$. Here $B_{n}$ denotes the $n$-th balancing number and $\pi(m)$ is the period of balancing numbers modulo any positive integer $m$. In this note, we establish some conditions related to the balancing-Wieferich primes.
\end{abstract}

MSC 2010. 11B25, 11B39, 11B41.

Key words. Balancing numbers, Wieferich primes, balancing-Wieferich primes, periodicity.

\section{REFERENCES}

[1] A. Behera and G. K. Panda, On the square roots of triangular numbers, Fibonacci Quart., 37 (1999), 98-105.

[2] R. Crandall, K. Dilcher and C. Pomerance, A search for Wieferich and Wilson primes, Math. Comp., 66 (1997), 443-449.

[3] F. Dorais and D. Klyve, A Wiefeich prime search up to $6.7 \times 10^{15}$, J. Integer Seq., 14, Article 11.9.2 (2011), 1-14.

[4] A.S. Elsenhans and J. Jahnel, The Fibonacci sequence modulo $p^{2}$-An investigation by computer for $p<10^{14}$, The On-Line Encyclopedia of Integer Sequences, 1-26.

[5] J. Klaška, Criteria for testing Wall's question, Czechoslovak Math. J., 58 (2008), 12411246.

[6] D. Marques, On the order of appearance of integers at most one away from Fibonacci numbers, Fibonacci Quart., 50 (2012), 36-43.

[7] D. Marques, The order of appearance of powers Fibonacci and Lucas numbers, Fibonacci Quart., 50 (2012), 239-245.

[8] D. Marques, The order of appearance of product of consecutive Lucas numbers, Fibonacci Quart., 51 (2013), 38-43.

[9] D. Marques, The order of appearance of product of five consecutive Lucas numbers, Tatra Mt. Math. Publ., 59 (2014), 65-77.

[10] R.J. Mcintosh and E.L. Roettger, A search for Fibonacci-Wieferich and Wolstenholme primes, Math. Comp., 76 (2007), 2087-2094.

[11] G.K. Panda, Some fascinating properties of balancing numbers, Congr. Numer., 194 (2009), 185-189.

[12] G.K. Panda and S.S. Rout, Periodicity of balancing numbers, Acta Math. Hungar., 143 (2014), 274-286.

The authors wish to thank the referees for useful comments and suggestions. P. K. Ray is the corresponding author.

DOI: $10.24193 /$ mathcluj.2018.1.05 
[13] B.K. Patel and P.K. Ray, The period, rank and order of the sequence of balancing numbers modulo m, Math. Rep. (Bucur.), 18 (2016), 395-401.

[14] P.K. Ray, Certain matrices associated with balancing and Lucas-balancing numbers, Matematika, 28 (2012), 15-22.

[15] S.S. Rout, Balancing non-Wieferich primes in arithmetic progression and abc conjecture, Proc. Japan Acad. Ser. A Math. Sci., 92 (2016), 112-116.

[16] Z.H. Sun and Z.W. Sun, Fibonacci numbers and Fermat's last theorem, Acta Arith., 60 (1992), 371-388

[17] D.D. Wall, Fibonacci series modulo m, Amer. Math. Monthly, 67 (1960), 525-532.

Received November 2, 2017

Accepted January 23, 2018

\author{
Sambalpur University \\ Department of Mathematics \\ Sambalpur, India \\ E-mail: utkaldutta@gmail.com \\ International Institute of Information Technology \\ Bhubaneswar, India \\ E-mail: iiit.bijan@gmail.com \\ Sambalpur University \\ Department of Mathematics \\ Sambalpur, India \\ E-mail: prasantamath@suniv.ac.in
}

